\title{
Werte beim frühen Carnap: Von den Anfängen bis zum Aufbau
}

\author{
A. W. Carus
}

In diesem Text ${ }^{1}$ skizziere ich einen Vorschlag zur Interpretation der geistigen Entwicklung Carnaps zwischen seinen Anfängen und der Niederschrift des Aufbau Mitte der Zwanzigerjahre. Ich behaupte, dass er von Anfang an einen bestimmten philosophischen Ansatz vertrat, den ich hier, um mich gegenwärtigem Gebrauch anzupassen, als „Funktionalismus“ bezeichnen werde. Ich wollte diesem hässlichen Wort aber keinen Platz in meinem Titel geben, und da es ja auch um Werte geht, habe ich Carnaps Auffassung der Werte stellvertretend für die anderen Sprachfunktionen in den Titel eingebracht.

„Funktionalismus“ heißt also bei manchen Philosophen die Idee, dass verschiedene Sprachebenen oder Sprachteile verschiedene Funktionen ausüben und daher nicht aufeinander reduziert werden können. Keiner dieser Teile ist als absoluter Maßstab in irgendeiner Hinsicht (z. B. was Sinn oder Bedeutung der Sprachausdrücke betrifft) für die anderen Teile zu betrachten. Die Kriterien der Wohlgeformtheit oder Zulässigkeit, die man bei einem Ausdruck einer bestimmten Sprachfunktion anwendet, sind im Allgemeinen nicht auf andere Sprachfunktionen anwendbar.

\footnotetext{
${ }^{1}$ Es wurde kein Versuch gemacht, den Stil des mündlichen Vortrags zu glätten, da es sich hier letztendlich nicht um eine umfassend belegte These handelt, sondern nur um einen vorläufigen Vorschlag zur Diskussion. Da können die zuweilen vielleicht übertrieben zugespitzten Formulierungen nur helfen, Reaktionen hervorzurufen. Ohne die Einladung der Herausgeber, Christian Damböck und Gereon Wolters, diesen Vortrag in Konstanz zu halten im Rahmen ihrer Konferenz über den jungen Carnap, wäre der Text nie zustande gekommen. Ich bin für die Diskussionsbeiträge dort sehr dankbar, vor allem für die kritischen Bemerkungen von Christian Damböck, Thomas Mormann, und Thomas Uebel, die zur Ausarbeitung der Thesen und Verbesserung der Formulierungen viel beigetragen haben.
}

\author{
A. W. Carus ( $ه)$ \\ Munich Center for Mathematical Philosophy, University of Munich, Munich, Deutschland
}

(C) The Author(s) 2021

C. Damböck, G. Wolters (eds.), Der junge Carnap in historischem Kontext:

1918-1935 / Young Carnap in an Historical Context: 1918-1935,

Veröffentlichungen des Instituts Wiener Kreis 30,

https://doi.org/10.1007/978-3-030-58251-7_1 
So allgemein dargestellt hat der Funktionalismus eine sehr lange Geschichte hinter sich, man denke etwa an Leibniz mit seiner Unterscheidung zwischen den verités de fait und den verités de raison sowie an Humes Unterscheidung zwischen „abstract reasoning concerning quantity or number“ und „experimental reasoning concerning matters of fact". Aber auch bei Kant und dem Neukantianismus behalten solche Unterscheidungen, wie z. B. die Unterscheidung zwischen deskriptiven und normativen Sätzen, entweder ontologische Anklänge oder (vor allem bei Hume und seinen Nachfolgern) psychologische. Erst mit der neuen Reflexion auf Sprache im 20. Jahrhundert, also mit Frege, Russell und Moore, und dann in reinerer Form mit Wittgenstein, wird Funktionalismus zu einem sprachphilosophischen Zugang zu all dem, was vorher die objektive Welt, objektive Wahrheiten der Mathematik, objektive Werte usw. ausgemacht hatte. Funktionalismus ist eine Konsequenz des ,linguistic turn" und bildet also, wenn man will, den Gegensatz zu allen ontologischen und platonistischen Ansichten über die objektive Existenz abstrakter (oder auch konkreter) Gegenstände, und zu jeder Art von Wertkognitivismus.

Carnaps Rolle in dieser Geschichte ist, dass er die Ansätze des späten 19. und frühen 20. Jahrhunderts bei Frege, Russell und Wittgenstein radikalisierte und von ihrer übrig gebliebenen ontologischen und platonistischen Restverschmutzung bereinigte und damit zum ersten Mal so etwas wie einen reinen und globalen Funktionalismus artikulierte - nicht klar genug und nicht ausführlich genug, das kann man schon zugeben. Aber dass er nicht weit genug gegangen wäre, wie Quine später monierte, und damit in die Metaphysik zurückgefallen wäre, kann man nicht behaupten. Im Gegenteil - Rick Creath (1994) hat gezeigt, dass Quine die funktionalistische Vorgehensweise Carnaps gar nicht begriff und dass seine Kritik an Carnap gerade aus diesem Missverständnis hervorgeht.

Ich möchte keineswegs behaupten, dass Carnap sein funktionalistisches Programm von Anfang an bewusst und in seiner ganzen späteren Reichweite konzipiert hätte. Nicht einmal in seiner späteren Entwicklung hat er dieses Programm, wie ich es hier definiere, ausdrücklich formuliert. ${ }^{2}$ In den frühen Jahren, von denen hier hauptsächlich die Rede sein wird, schwebte es ihm gewiss nur als relativ vage Idee vor. In den folgenden Ausführungen zeigt sich allerdings, dass dieser noch verschwommene, unklare Proto-Funktionalismus sehr fest in ihm verankert war und dass er diese quasi-instinktive Ausgangsposition mit einer gewissen schlafwandlerischen Sicherheit konsequent immer klarer artikulierte und inhaltlich immer weiter entwickelte.

Diese Ausgangsposition muss, um sie richtig einordnen zu können, vor dem Hintergrund des damals unter Naturwissenschaftlern weit verbreiteten Positivismus gesehen werden, in dem die (später von Carnap so genannte) kognitive Sprachfunktion meist als einzig legitime anerkannt wurde. Mills versuchte Darstellung der Mathematik als System sehr allgemeiner empirischer Sätze wurde z. B. weithin akzeptiert, wie auch der Utilitarismus oder andere versuchte positivistische und naturalistische Reduktionen der Werte, wie etwa bei Ostwald. ${ }^{3}$

\footnotetext{
${ }^{2}$ Hierzu siehe meine Ausführungen in Carus (2007) sowie in Carus (2017), z. B. S. 19.

${ }^{3}$ Ostwalds ,energetische“ Ableitung der Werte aus dem Zweiten Hauptsatz der Themodynamik wird selbst im Aufbau (Carnap 1928, § 59) noch anerkennend erwähnt.
} 
Die frühe analytische Philosophie zeichnete sich gleich zu Anfang dadurch aus, dass sie diese Beschränkung in zweierlei Hinsicht überwand - in der Mathematik durch Frege und in der Wertphilosophie durch Moore. Frege hatte bekanntlich schon in seinen Grundlagen die empiristische Assimilierung der Mathematik an den empirischen Bereich (wie bei Mill) vernichtend widerlegt. Aber erst Wittgenstein gelang es, im Tractatus, die platonisch-ontologische Deutung dieser Funktionsdifferenzierung loszuwerden, indem er die Logik als Nebenprodukt der bildlichen Darstellung der Welt in Sätzen umdeutete. Er war allerdings kein Logizist im selben Sinne wie Frege oder Russell, aber er sah die Mathematik in ähnlichem Sinne, und der Wiener Kreis war so glücklich, die platonischen Verwicklungen der Mathematik loszuwerden, dass er die Aufgabe gern in Kauf nahm, den Tractatus logizistisch umzudeuten und seine finitistischen Aspekte mit der klassischen Mathematik und der theoretischen Naturwissenschaft zu versöhnen. Dies sah Carnap jedenfalls in den ersten Wiener Jahren als seine Hauptaufgabe an. ${ }^{4}$

Die normative Sprachfunktion wurde im funktionalistischen Sinne von der kognitiven losgelöst bei Moore (in den Principia Ethica), indem er langwierig, und nie sehr klar, zu zeigen versuchte, dass ,gut“ kein deskriptives Prädikat im selben Sinne wie ,gelb“ sein kann. Jedenfalls ging die ganze Diskussion über das, was Carnap im Nachhinein „Nonkognitivismus“ nannte, vor allem in England, mit Moore, los - in einer Tradition, mit der Carnap erst sehr viel später in Berührung kam. Diese Diskussion beschränkte sich nicht auf die Kreise der von Moore maßgeblich beeinflussten Ordinary-language-Philosophen, sondern fand auch Anklang auf der anderen Seite des Atlantik, vor allem bei Charles L. Stevenson, der dann wiederum in England gelesen und verwertet wurde. Richard Hare (1952), ein Schüler von John Austin wie viele andere Teilnehmer dieser Diskussion, nahm diese verschiedenen Impulse auf und artikulierte in den Fünfzigerjahren, was man wohl als klassische Ausführung des ethischen Nonkognitivismus in der Folge von Moore bezeichnen darf.

Aber schon in der nächsten Generation fand sich Hare der Kritik preisgegeben, dass er der ordinary language nicht treu genug geblieben war. Bernard Williams (1985) zum Beispiel, sein Nachfolger in Oxford, meinte ganz richtig, dass Hares strenge Unterscheidung von deskriptiver und normativer Sprache in der Umgangssprache selbst nicht aufzufinden war, sondern als künstliches Konstrukt in sie importiert werden müsste. Diese Kritik trifft Hare empfindlich, denn rhetorisch zumindest hatte er seine Logik der normativen Sprache in The Language of Morals aus umgangssprachlichen Motiven und Unterscheidungen entwickelt. Wenn man den Text genauer anschaut, fällt seine Zweideutigkeit in dieser Hinsicht ins Auge, aber Hare konnte nicht anders als - seiner akademischen Herkunft getreu - so zu

\footnotetext{
${ }^{4}$ Awodey und Carus (2001) belegen dies im Einzelnen. Carnap blieb seit 1926 hartnäckig an seinem Versuch, Wittgensteins funktionalistische Interpretation der Logik und Mathematik irgendwie mit der klassischen Mathematik und der theoretischen Physik zu vereinen, und deshalb genügte ihm Hilbert philosophisch nicht. Er versuchte, um jeden Preis Wittgenstein mit Hilbert zu kombinieren, vor allem in seinem bemerkenswerten Entwurf einer „Neuen Grundlegung der Logik“ von 1929. Und diese Untersuchungen führten ihn, wie Awodey und Carus $(2007,2009)$ auch beschrieben haben, gegen Ende 1932 letztlich zum Toleranzprinzip. Hiermit ist der Weg frei zu eben einem solchen globalen und pluralistischen Funktionalismus, wie ihn Huw Price neuerdings wieder betreibt (worauf im Folgenden eingegangen wird).
} 
tun, als berufe er sich auf eine funktionale Unterscheidung, die in der Umgangssprache selbst verwurzelt ist und somit eine objektive Komponente der „Realität“ darstellt. Denn, wie Peter Strawson (1963, S. 518), Hares Kollege in Oxford und Mitzögling des Austin-Kreises, das Grundprinzip der Ordinary-language-Schule in seiner Kritik an Carnap ausdrückt: „,... the actual use of linguistic expressions remains [the philosopher's] sole and essential point of contact with reality. “

Carnap hat dieses Problem nicht; das Grundvorurteil der Ordinary-languageSchule hatte er nie geteilt. Im Gegenteil - ein globaler Funktionalismus kann sich nicht um ,contact with reality“ kümmern. Und er kann die Umgangssprache nicht als universellen Maßstab betrachten. ${ }^{5}$ Denn in der Umgangssprache ist alles verwoben und schwer unterscheidbar; eine umgangssprachliche Aussage kann (empirisch gesehen) zugleich mehrere Funktionen haben, die nicht klar unterscheidbar oder begrifflich getrennt sind. Ein globaler Funktionalismus setzt also voraus, dass die einzelnen „Funktionen“ der Sprache bewusst bestimmt und explizit formuliert werden - dass sie, mit anderen Worten, gemacht werden und nicht schon irgendwo in der sprachlichen (oder psychologischen oder neurologischen) Natur vorzufinden sind. (Das war ja der ganze Sinn der Kampagne gegen die „Metaphysik“ - es ging nicht darum, irgendwelche Fragen auszugrenzen, sondern die Forderung abzulehnen, dass uns die Funktionen der Sprache oder die Grenzen zwischen ihnen irgendwie letztlich vorgegeben sind, also von uns entdeckt oder aufgefunden werden könnten.)

Nach der Kritik an Hare ging der klassische Nonkognitivismus in der Nachfolge von Moore eine Zeit lang in die Defensive, wurde dann aber unter dem neuen Namen „Expressivismus“ von Simon Blackburn und Alan Gibbard wieder aufgegriffen. In jüngster Zeit hat Huw Price (1988), in Anschluss an Blackburn, diesen Expressivismus $\mathrm{zu}$ verallgemeinern versucht und einen „globalen Expressivismus“ angestrebt. Dabei beruft er sich interessanterweise auf Carnap (Price 1997), und zwar auf Carnaps funktionalistische Deutung der Logik und Mathematik - die auch Rick Creath (1994) in seinem Funktionalismus-Aufsatz vor allem im Sinn hat, wenn er Quines Unverständnis dieses Funktionalismus anprangert. ${ }^{6}$

Ich möchte jetzt unter diesem allgemeineren Gesichtspunkt des minimalistischen Funktionalismus, wie er Price vorschwebt (siehe auch Carus 2017), untersuchen, ob es in Carnaps früher Entwicklung irgendwo einen Bruch gibt, eine Diskontinuität in dieser Hinsicht. Dass es einen solchen Bruch, oder mehrere solche Brüche, gegeben haben soll, wird ja bekanntlich von Thomas Mormann $(2006,2016)$ argumentiert merkwürdigerweise auch (zumindest implizit) von Carnap selbst, der im Schlussteil seiner Autobiographie sagt: „I have maintained the thesis [of the non-cognitive nature of value statements] for about thirty years" (Carnap 1963, S. 82). Wenn man an-

\footnotetext{
${ }^{5}$ Carnaps Ablehnung der Ordinary-language-Schule wird in seiner Autobiographie kurz angesprochen (Carnap 1963, S. 68) und in seiner Replik auf Strawson (ebd., S. 933-940) ausführlicher begründet; siehe in diesem Zusammenhang auch Carus (2018), S. 450-454.

${ }^{6}$ Die Beziehungen zwischen Carnap und Price habe ich in Carus (2018) genauer darzustellen versucht - mit weitgehender Zustimmung seitens Price' (2018) selbst in seiner Replik.
} 
nimmt, das er diesen Satz nicht revidiert hat, als sich die Veröffentlichung des Schilpp-Bandes jahrelang verzögerte, müsste man seine Aussage also auf Mitte der Fünfzigerjahre datieren, und den Zeitpunkt seiner angeblichen Bekehrung zum Nonkognitivismus auf etwa 1925.

Als Nullhypothese nehme ich aber die Annahme, dass Carnap, falls er hier auf eine erhebliche Diskontinuität hinweisen wollte, im Unrecht über seine eigene Entwicklung ist. (Was übrigens ihn selbst, der sich immer über sein äußerst schlechtes Gedächtnis beschwerte, nicht überrascht hätte. $)^{7}$ Und das Resultat - was hätten Sie anderes von mir erwartet? - ist natürlich, dass sich diese Nullhypothese auch bewährt. Um das zu zeigen, bräuchte man viel mehr Material, als ich in diesem kurzen Vortrag besprechen kann. Wir gehen aber mal der Reihe nach einige Stationen von Carnaps früher Entwicklung durch, bis zum Aufbau, und schauen, ob sie in der einheitlichen Weise, die ich vorschlage, gedeutet werden können.

\section{1 „Religion und Kirche“ (1911)}

Als erstes Dokument verweise ich auf Carnaps Notizen für eine Rede, die er 1911 vor der neugegründeten Freiburger Freischar hielt. ${ }^{8}$ „Religion und Kirche“ heißt dieser Vortrag, und sein Sinn bestand vor allem darin, eine bestimmte Hauptunterscheidung klarzumachen und dann mit vielen historischen Beispielen zu veranschaulichen. Carnap beginnt mit der Aufstellung von drei Hauptbestandteilen der Religion:

1. dem Kultus, oder Handlungen, um eine Gesinnung gegenüber dem Höheren auszudrücken,

2. der Ethik, oder der Gesinnung gegenüber anderen Menschen und der Gesellschaft, die aus der Gesinnung gegenüber dem Höheren resultiert, und

3. den Lehrsätzen, die eine Gesinnung in Worte fassen sollen. Carnap nimmt diesen Lehrsätzen gegenüber eine sehr entschieden skeptische Haltung ein: „,die Religion besteht nicht nur nicht in den Lehrsätzen, - was jeder zugeben wird, - sondern sie kann durch sie weder unterstützt noch gestürzt werden, da sie von ihnen überhaupt nicht berührt wird“ (ASP 081-47-05, S. 5).

Man könnte allerdings entgegnen, sagt Carnap, dass ,jede Religion doch mit Hülfe des Wortes von Mensch zu Mensch weitergegeben wird“ (ebd.). Da muss

\footnotetext{
${ }^{7}$ Ein Beispiel: Als Carnap 1955 zum ersten Mal seine Idee der Heraustrennung des empirischen Gehalts eines theoretischen Satzes durch den Ramsey-Satz vortrug, hatte er völlig vergessen (wie er später in einem Brief an Hempel selbst amüsiert bemerkte), dass er diese Idee nicht nur vor Jahren in Ramseys Text gesehen, sondern die relevante Stelle sogar unterstrichen hatte (Psillos 2000, S. 153, Fußnote 7)!

${ }^{8}$ Dieser Vortrag wird demnächst in Damböck et al. (2019) veröffentlicht werden, zusammen mit meinem Vortrag in dieser Konferenz (über „Carnap und die Religion“), wo Carnaps Vortragsnotizen von 1911 ausführlicher kommentiert werden.
} 
man jedoch, sagt er, unterscheiden zwischen zwei Arten von Lehrsätzen, und hier liefert er dann seine Hauptunterscheidung, die den ganzen übrigen Vortrag in Anspruch nimmt:

Unter den Sätzen, die allerdings zu diesem Zwecke gesprochen werden müssen, möchte ich deshalb unterscheiden zwischen denen, die sich auf verstandesmäßig erfaßbare Dinge beziehen, und denen, die ethische Forderungen oder die subjektive Auffassung des Weltganzen und des Menschenlebens zum Ausdruck bringen. Die erstere Art von Behauptungen, nämlich die über ihrer Natur nach objektive, wenn auch vielleicht noch nicht erkannte oder unerkennbare, Thesen, will ich „Wissenssätze" nennen. Alle nur möglichen menschlichen Wissenssätze bilden in ihrer Gesamtheit das „Weltbild“. Ihnen gegenüber stehen die Sätze über unsere Stellung zu einem Weltbild, die also beispielsweise unseren Pessimismus oder Idealismus oder Realismus zum Ausdruck bringen; sie lassen sich weder rein verstandesmäßig beweisen, noch widerlegen, also nicht diskutieren. Ich will sie „Glaubenssätze“ " nennen ... (ebd.)

Der darauffolgende historische Exkurs mit den vielen Beispielen, soll zeigen, dass keine „Religion“ in Carnaps Sinn (wozu auch die Weisheitslehren der antiken philosophischen Schulen usw. gehörten) von „Wissenssätzen“ abhing oder von ihren Gründern und Stiftern als davon abhängig betrachtet wurde.

Es ging Carnap in diesem Vortrag also vor allem darum, jede Form von Religion oder Gesinnung, einschließlich der nicht-religiösen Ethik (wie wir gleich sehen werden), so radikal wie möglich von jeglichem propositional-deskriptiven Wissen abzutrennen: ,die Religion besteht nicht nur nicht in den Lehrsätzen, ... sondern sie kann durch sie weder unterstützt noch gestürzt werden, da sie von ihnen überhaupt nicht berührt wird" (meine Hervorhebung).

Außerdem macht Carnap klar, dass er unter „Religion“ nicht den Glauben an etwas Außer- oder Übernatürliches versteht, sondern eine gewisse Funktion im Wertehaushalt; unter Religion versteht er eben dasjenige, was als übergeordneter Wert funktioniert, der alle anderen Werte im Wertehaushalt des Einzelnen (oder einer wertehomogenen Gruppe) bestimmt:

Ich fasse hier Religion weiter, als es gewöhnlich geschieht. Ich sehe sie als etwas allgemein menschliches an, was weder von dem Glauben an einen Gott, - wie ich ja bisher überhaupt noch nicht von irgendeinem Glauben in diesem Sinne gesprochen habe, - noch etwa an ein bestimmtes Ideal abhängig wäre. So ist nach meiner Auffassung z. B. auch der Patriotismus Religion und seine Betätigung Religionsausübung, nämlich für den Menschen, dem das Vaterland auf der höchsten Stufe seiner Wertung steht. Was für den Menschen auf dieser Stufe steht, ist für die Frage, ob sein Verhältnis dazu Religion ist oder nicht, prinzipiell gleichgültig, wenn wir auch zuweilen an anderen Menschen eine solche Religion höher als eine andere werten. Während dem einen auf der Stufe des höchsten Wertes ein persönlicher Gott steht, oder das Weltganze als Organismus im pantheistischen Sinne, stellt z. B. ein anderer dorthin die Kunst im allgemeinen, oder eine bestimmte Kunst, oder die Wissenschaft; wieder andere Familie, Vaterland, Rasse, Menschheit. (ebd., S. 3-4)

Schon der 19- oder 20-jährige Carnap vertrat also sehr bewusst einen betonten Funktionalismus (oder vielleicht Proto-Funktionalismus) bezüglich der Sprache der 
übergeordneten Werte, ${ }^{9}$ und zwar nicht nebenbei, sondern als Hauptanliegen seines Vortrags; die Frage hat ihn offenbar in seinen frühen Jahren stark beschäftigt, und er hat viel darüber nachgedacht.

Es drängt sich natürlich die Frage auf, woher das beim jungen Carnap denn gekommen sein mag. Der Zusammenhang dieses Freischar-Vortrags legt nahe, dass die Beschäftigung mit Religion eine große Rolle, wenn nicht die Hauptrolle, dabei spielte. Wir wissen ja, dass Carnap sehr religiös erzogen wurde, dass aber theologische „Wissenssätze“ bei der Religiosität der Eltern höchstens eine sehr untergeordnete Rolle spielten (Carus 2007, 1. Kapitel). Carnap beruft sich in seinem Vortrag zudem mehrfach auf die ,neuere Theologie“ der damals viel gelesenen Johannes Müller, Heinrich Lhotzky und Christoph Schrempf, deren Lehren auf gerade diese scharfe Trennung von „Glaubenssätzen“ und „Wissenssätzen“ hinausliefen. Sie gingen über Entmythologisierung hinaus und sahen den Wert der Religion vor allem in der Berührung mit dem Höheren, was das nun immer im einzelnen Fall oder für den einzelnen Menschen sein mag; die Doktrin sollte möglichst beiseite gelassen werden. Sehr ähnlich war übrigens die religiöse Einstellung von Eugen Diederichs, in dessen Sera-Kreis Carnap sich in diesen Jahren sehr aktiv beteiligte, wie man im relevanten Kapitel von Meike Werners Buch über den Sera-Kreis (Werner 2003) nachlesen kann.

Die strikte Trennung von „Wissenssätzen“ und „Glaubenssätzen“ erlaubte es Carnap also in diesen Jahren (bis nach dem Krieg sogar), nominell am Glauben seiner Eltern festzuhalten und sich nicht von der Kirche zu trennen, trotz wachsender Skepsis gegenüber den kirchlichen Lehren.

\subsection{Brief an LeSeur (1916)}

Fünf Jahre später hatte sich viel geändert. Es ist nun Krieg, Carnap sitzt im Schützengraben an der Front, wo er an einigen der blutigsten Kämpfe beteiligt ist. Religion ist immer noch im Vordergrund, aber die Haltung ist noch um einiges ablehnender geworden. Nun sind es nicht mehr nur die „Wissenssätze“ der Kirche, die von Carnap abgelehnt werden, sondern auch die „Glaubenssätze“, die Einstellung zum Leben, die von der Kirche gefordert wird. ${ }^{10}$

\footnotetext{
${ }^{9}$ Mancher wird vielleicht einwenden, man könne doch nicht den späteren „linguistic turn“ des Tractatus schon dem frühen Carnap zuschreiben. In seiner ganzen Tragweite gewiss nicht; andererseits muss anerkannt werden, dass Carnap in seinem Vortrag selbst betont, nicht von „Glauben“ oder „Wissen“ sprechen zu wollen, sondern von (möglichen) Ableitungsbeziehungen zwischen Glaubenssätzen und Wissenssätzen. Insofern bezieht sich Carnap hier schon auf sprachliche Darstellung der Welt statt auf eine „Wirklichkeit“ hinter dieser Darstellung.

${ }^{10}$ Carnap trat offiziell erst nach Kriegsende aus der Kirche aus, erwog aber den Austritt offenbar schon im Zusammenhang dieses offenen Briefes in Diskussionen mit seiner Mutter; siehe meine Einleitung zur Veröffentlichung des Briefes in Damböck et al. (2019). Bei seiner Heirat mit Elisabeth Schöndube 1917 zeigt auch seine Korrespondenz mit ihr und seiner Mutter, dass er lieber auf
} 
Bei dem zweiten hier diskutierten Dokument handelt es sich um einen offenen Brief gegen einen gewissen Berliner Pastor Eduard LeSeur, der in einem Artikel (LeSeur 1916) behauptet hatte, dass, da die moderne Kultur offenbar zu schwach gewesen war, die Katastrophe des Krieges zu verhindern, sie in den Hintergrund treten sollte, zugunsten einer Erneuerung des traditionellen christlichen Glaubens. ${ }^{11}$ Unter anderem hatte LeSeur gemeint, dass nur das Christentum dazu imstande sei, eine Lücke zu finden im drohenden Determinismus der Naturwissenschaften. Worauf Carnap entgegnet:

$\mathrm{Zu}$ ihm, so glauben Sie, muß uns das erschreckende Bewußtsein des Determinismus hinführen. „Die Erkenntnis dieser unbedingten Unfreiheit führt zur Verzweiflung.“ „In diese gebundene Menschheit hinein stellt Gott den Christus Jesus." Aber ich stehe nur als natürliches Wesen in dem Kausalnexus. Als ethisches Wesen bin ich selbst dagegen frei entscheidendes Subjekt meiner Handlungen. ,Jesus bahnt der Freiheit derer, die ihm folgen, eine Gasse." Also auch Sie sind jetzt frei und selbst entscheidendes Subjekt, wenn auch nach Ihrem Glauben durch die Hilfe eines andern Wesens. (ASP 089-74-01, S. 17-18)

Carnap hat sich also in der Zwischenzeit die Kantische Auffassung der Beziehung zwischen den sensiblen und den intelligiblen Aspekten der Welt angeeignet, d. h. die Unterscheidung zwischen dem Theoretischen und dem Praktischen. Alan Richardson (2007) sieht in dieser Unterscheidung den Ursprung von Carnaps eigener lebenslanger Unterscheidung zwischen theoretischen und praktischen Diskursen, also den Ursprung seines Nonkognitivismus. Nach der Lektüre des Vortrags von 1911 können wir aber definitiv sagen, dass diese Unterscheidung bei Carnap weiter zurückgeht als ihre Artikulierung in Kantischer Sprache.

Trotz dieser Einbettung in Kantische Formen blieb Carnaps Nonkognitivismus fünf Jahre nach seinem Freiburger Vortrag unvermindert bewusst und radikal. Wenn er z. B. LeSeurs religiösen Weg schroff ablehnt und sogar meint, er sei gar kein Weg, sondern nur „die Art, wie Sie und die Menschen Ihres Glaubens das innere Suchen nach einer Antwort auf jene Frage zur Ruhe gebracht haben“, fügt er hinzu: „Theoretisch läßt sich hierüber ja nichts sagen“ (ebd., S. 12). Es handelt sich schließlich, wie er sich 1911 ausgedrückt hatte, um „Glaubenssätze“, nicht um „Wissenssätze“ - es handelt sich um Gesinnung, nicht um Wissenschaft.

Das heißt aber natürlich eben nicht, dass Gesinnungen nicht rational diskutierbar sind. Gleich nach dem eben zitierten Satz, ,,Theoretisch läßt sich hierüber ja nichts sagen“, geht der Text des offenen Briefes nämlich mit der folgenden Argumentation weiter:

Aber es genügt, wenn die Allgemeingültigkeit des Weges dadurch hinfällig wird, daß er für mich nicht gangbar ist; selbst wenn es nicht all die anderen Menschen, die so sind wie ich, noch gäbe. Ja, werden Sie denken, das ist das „Eisengitter“. Doch wir stehen uns nicht in so entgegengesetzten Richtungen gegenüber, wie Sie vielleicht erwarten. Ich will einmal mög-

die kirchliche Trauung verzichten wollte, als dass ein traditioneller Geistlicher das Amt dabei übernahm (zum Glück ließ sich in letzter Minute ein offenbar genügend aufgeklärter finden).

${ }^{11}$ Auch dieses Dokument wird im oben genannten Konferenzband (siehe Fußnote 10) veröffentlicht werden. 
lich[st] nahe zu Ihrem Standpunkt herantreten. Dann gerade wird sich am deutlichsten die unüberwindbare Kluft zeigen, die dann noch zwischen uns bestehen bleibt. (ebd., S. 12-13)

Das ist ein wenig paradox ausgedrückt: Erstens kann man theoretisch hierzu nichts sagen, zweitens lässt sich doch noch was Theoretisches sagen, nämlich dass ein einziges Gegenbeispiel die Allgemeinheit des optativen Satzes von LeSeur hinfällig macht, drittens dass dies nicht zeigt, wie LeSeur daraufhin vielleicht sagen würde, dass hier eine unüberbrückbare Kluft besteht zwischen Carnaps Standpunkt und seinem eigenen, viertens dass Carnap jetzt versuchen wird, seinen Gesinnungsstandpunkt in einer Weise darzustellen, die LeSeurs Standpunkt so ähnlich wie möglich aussieht. Aber letztens, dass gerade diese Darstellung die ,unüberwindbare Kluft" deutlich aufzeigen wird, ,die noch zwischen uns besteht“. Also lässt sich doch, trotz der ausdrücklichen Ablehnung, ,theoretisch einiges hierüber sagen“!

Diese letzten Schritte würde ich etwa so deuten, dass Carnap hier sagen will: „Die Unterschiede zwischen uns sind nicht so radikal, wie Sie sich das vielleicht denken, und ich werde jetzt meinen Standpunkt so beschreiben, dass man ihn zu Ihrem in Beziehung bringen kann, also so, dass die Parallelen zwischen unseren Gesinnungen klarer hervortreten - was uns gleichzeitig auch die noch bestehenden Unterschiede klarer machen wird." So eine Vorgehensweise wäre ja sehr charakteristisch für den späteren, philosophischen Carnap, der immer zu präzisieren versuchte (oft mit mehrspaltigen Tabellen), wo nun genau der Unterschied zwischen scheinbar unüberbrückbaren Standpunkten zu lokalisieren sei. Wie aber ist eine solche Argumentation überhaupt möglich, in Hinblick auf das schroffe ,Theoretisch läßt sich hierüber ja nichts sagen“" und vor allem auf Carnaps Charakterisierung im Freiburger Vortrag, die Glaubenssätze brächten „unsere Stellung zu einem Weltbild ... beispielsweise unseren Optimismus oder Pessimismus oder Realismus zum Ausdruck ...; sie lassen sich weder rein verstandesmäßig beweisen, noch widerlegen, also nicht diskutieren"? Hat sich hier also in fünf Jahren doch etwas geändert?

Ich glaube nicht. Ich denke, man muss die Argumentation gegen LeSeur dahingehend verstehen, dass rein auf der Ebene der Theorie selbst, also rein verstandesmäßig, zwar nichts gesagt werden kann - aber dass man schon Argumente vorbringen kann für und gegen Glaubenssätze, und zwar nicht nur theoretische, verstandesmäßige Argumente in einer ergänzenden Aushilfefunktion, sondern vor allem normative Argumente der praktischen Vernunft, oder (wie sich Carnap vierzig Jahre später, in einem Fragment von 1958, ausdrückte) ,purely valuational criteria by which to judge a value function as more or less rational than another" (Carnap 2017, S. 193).

Das mutet vielleicht wie ein großer Sprung an, über ein halbes Jahrhundert hinweg. Aber wir werden im folgenden Abschnitt sehen, wie Carnap - vermutlich in genau diesem Sinne einer praktischen Rationalität - zwei Jahre nach dem LeSeurBrief von „objektiven Werten“ spricht. Und Richardson (2007) weist darauf hin, dass die zentrale Doktrin Carnaps in seiner mittleren und späten Zeit, das Toleranzprinzip, überhaupt keinen Sinn hätte, wenn man dem Theoretiker (dem conceptual engineer oder der scientific community) nicht die praktische Freiheit der Wahl zwi- 
schen Sprachrahmen unterstellte. Richardson zitiert aus dem Aufsatz „Theoretische Fragen und Praktische Entscheidungen“" (Carnap 1934), um zu zeigen, dass sich Carnap diese Wahl zwischen Sprachen auch in der extremsten Syntax-Zeit nicht als willkürlich oder irrational vorstellte. Er meinte bloß, damals wie in den früheren Schriften von 1911 und 1916 sowie in dem späteren Fragment von 1958, dass die Argumente für und wider bestimmte Wertsätze nicht rein verstandesmäßig sein konnten; logischen und empirischen Argumenten kommt in diesem Zusammenhang nur eine Hilfsfunktion zu. Argumente der praktischen Rationalität mussten also aus den ,purely valuational criteria by which to judge a value function as more or less rational than another" bestehen oder wesentlich von solchen Kriterien abhängen.

\section{3 „Deutschlands Niederlage“ (1918)}

Im folgenden Jahr, fast genau zur Zeit der deutschen Revolution und des Kriegsendes, benutzt Carnap in einer ,politischen Stellungnahme“, dem Aufsatz „Deutschlands Niederlage“ für Karl Bittels Politische Rundbriefe (der aber nicht veröffentlicht wurde), ${ }^{12}$ den Begriff der ,praktischen Vernunft“ auch explizit, und ich sehe keinen Grund, dieses Dokument nicht im selben Kantischen Sinne wie den zeitnahen Brief an LeSeur zu interpretieren. Keine Textstelle darin ist mit dieser Auslegung unvereinbar. Die gemeinsamen Werte der Leserschaft der Politischen Rundbriefe, auf die sich Carnap wiederholt ausdrücklich beruft, werden zwar als vernunftbegründet angesehen, aber begründet offenbar durch praktische Vernunft und es wird klar gemacht, dass sie die Werte einer bestimmten Gruppe sind. Nirgends wird behauptet, diese „objektiven Werte“ würden objektiv für alle Menschen gelten. Und nur nach einem gegebenen Maßstab (in diesem Fall dem der demokratisch gesinnten Anhänger der Vorkriegsjugendbewegung) kann von „Fortschritt“ oder „Rückschritt“ bei Werten gesprochen werden. Bei Kant ist ja der Begriff der Vernunft ein weiterer als der des Verstandes; der Verstand bezieht sich auf das Sensible, die Vernunft auf das Sensible und das Intelligible insgesamt. Dass „Vernunft“ hier - wie bei Kant üblich - auf beides angewandt wird (obwohl eher beiläufig), sehe ich also im Gegensatz zu Mormann (2010) nicht als Grund, eine Abkehr von seinem vorherigen Funktionalismus in Carnaps Text hineinzulesen und ihn nun in einen Anhänger Rickerts zu verwandeln.

Obwohl in „Deutschlands Niederlage“ tatsächlich von „objektiven Werten“ die Rede ist - was zugegebenermaßen schon sehr nach Rickert klingt -, muss man außerdem Carnaps Wendigkeit in Bezug auf die verschiedenen philosophischen „Sprachen“ in Betracht ziehen, die er angeblich mit den Anhängern verschiedener Schulen zu sprechen gewohnt war. Bei Kant selbst könnte man ja durchaus auch von „objektiven Werten“ sprechen (obwohl er diese Vokabel nicht verwendet), und es

\footnotetext{
${ }^{12}$ Dieses Dokument wird eingehend besprochen von Carus (2007), Kap. 1; Mormann (2010) gibt eine stark abweichende Interpretation, ohne aber auf meine Darstellung einzugehen (oder sie auch nur zu erwähnen). Auch dieses Dokument erscheint in Damböck et al. (2019).
} 
gibt im Artikel von 1918 keine Anhaltspunkte, etwas Weitergehendes hinter dieser Wortwahl zu suchen.

Ich möchte natürlich nicht behaupten, dass Rickert, dessen Vorlesungen über Goethe (und wohl andere Themen) Carnap in Freiburg begeistert besucht hatte, ohne jeden Einfluss auf ihn geblieben ist. Im Vortrag von 1911 wird Rickert beiläufig erwähnt, aber man würde nie darauf kommen, diesen Vortrag dem SüdwestNeukantianismus zuzuordnen. Im Aufbau wird Rickert tatsächlich mehrmals erwähnt, aber das dient, wie ich im 5. Abschnitt behaupten werde, vor allem der Ablehnung seiner Philosophie. Dort werden wir auch auf „Deutschlands Niederlage“ wieder zurückkommen, wo der ganzen akademischen Tendenz, der Rickert angehörte (dem Historismus), ein guter Teil der Kriegsschuld zugewiesen wird. ${ }^{13}$

\section{4 „Vom Chaos zur Wirklichkeit““ (1922) und der Umbau von 1924}

Nun kommen wir zum Aufbau-Projekt. Dieses geht eigentlich weiter zurück als 1922, wie verschiedene Notizen aus früheren Jahren zeigen; aber die Lektüre des Buchs Our Knowledge of the External World von Russell war das große DurchbruchErlebnis für Carnap. Es schlug sich dann auch innerhalb weniger Monate im Manuskript „Vom Chaos zur Wirklichkeit“ nieder ${ }^{14}$ obwohl der Einfluss Russells in der Einleitung zu diesem Dokument nicht zu spüren ist. Einige (wie Mormann) neigen deshalb auch hier wieder dazu, Rickert hinter der Szene zu wittern. Ich würde zwar wie vorher zugeben, dass das Vokabular in „Von Chaos zur Wirklichkeit“ an manchen Stellen zweideutig ist, aber man sollte wirklich nicht nur aufs Atmosphärische schauen, sondern darauf, was hier gesagt wird. Das Hauptanliegen dieser Einleitung ist ganz offenbar, einen pragmatistischen Zugang zu einem sinnvollen und allgemein zumutbaren Ausgangspunkt des Konstitutionssystems zu bahnen; Carnap will so wenig voraussetzen wie möglich. Er gibt also gleich zu Anfang unumwunden zu, dass die ganze Konstruktion der Wirklichkeit aus einem Empfindungschaos eine Fiktion darstellt (ein Wort, das er auf diesen paar Seiten so oft wiederholt, dass man nicht umhin kann, einen Einfluss Vaihingers zu vermuten), was ja die Anklänge an Schopenhauer und Nietzsche erklären würde (die Mormann auch nicht entgehen). Vaihinger tendierte bekanntlich zu einem gewissen eigenartigen Pragmatismus (er selbst räumte die Ähnlichkeit durchaus ein); ich würde also den quasi-pragmatisti-

\footnotetext{
${ }^{13}$ Über dieses Dokument wäre viel mehr zu sagen, als in diesem engen Raum möglich ist. Da Mormanns (2010) Interpretation von meiner so grundverschieden ist, müsste man den ganzen $\mathrm{Zu}$ sammenhang im Einzelnen erörtern. Eine solide Grundlage hierfür bildet neuerdings Werner (2015), wo Carnaps politische Gedankenwelt von 1918 ausführlich dokumentiert wird.

${ }^{14}$ ASP 081-05-01; siehe hierzu Carus (2007, Kap. 5), mit längeren Zitaten aus dem Typoskript und detaillierten Archivhinweisen. Carnap las Russells Buch im Winter 1921-22; „Vom Chaos zur Wirklichkeit“" entstand im Juni 1922.
} 
schen Einschlag beim frühen Carnap, auch im Aufbau, zumindest teilweise dem Einfluss Vaihingers zuschreiben. ${ }^{15}$

Festzuhalten ist, dass dieser fiktionalistisch-pragmatistische Zugang zum Ausgangspunkt des Konstitutionssystems in der ersten Phase des Aufbau-Projekts sich nahtlos in die Entwicklung der funktionalistischen Perspektive einfügt, an die sich Carnap schon 1911, 1917 und 1918 herantastet. Die Sprache, die Mathematik, die Wissenschaften werden zunehmend als Werkzeuge betrachtet, die wir zu bestimmten menschlichen Zwecken so einrichten, wie es diesen Zwecken entspricht. Im Fall des frühen Aufbau-Projekts betrifft es nicht die Sprache der Werte, sondern die Sprache der empirischen Erkenntnis, die bei Carnap jetzt in den Vordergrund rückt (und einige Jahre dort bleibt, bis ab etwa 1927 die Logik und Mathematik diese Stellung übernehmen). Aber dieser Wechsel des Aufmerksamkeitsbrennpunkts darf nicht von der fortschreitenden Entwicklung des funktionalistischen Grundmotivs ablenken, das sich quer durch die wechselnden Anwendungsgebiete in diesen Jahren immer weiter verfestigt und tiefer dringt.

Zwei Jahre nach dem ersten Ansatz hat Carnap das ganze Aufbau-Projekt nochmals grundlegend umgebaut. Ich möchte hier nur auf drei Punkte hinweisen, die im Wesentlichen auch von Carnap selbst, nach dem Umbau, als seine neuen Hauptthesen aufgestellt werden, bei der Vorstellung der Konstitutionstheorie in Wien im Januar 1925 (ASP 081-05-02):

1. Ablehnung der Ontologie

2. Einheit des Gegenstandsbereichs

3. Überwindung der Subjektivität

Zum ersten Punkt: Irgendwann im Laufe des Jahres 1924 warf Carnap das ursprüngliche Hauptwerkzeug für die Aufstellung der Basis seiner Konstitutionstheorie über Bord - die Phänomenologie. Vorher hatte es eine primäre Welt der unmittelbaren Sinneseindrücke gegeben, der Carnap durch phänomenologische Wesensschau genügend Struktur geben konnte, dass die Methode der Quasianalyse auf sie angewandt werden konnte, um sekundäre Welten (oder „Wirklichkeiten“) auf der feststehenden, gegebenen primären Welt aufzubauen. Ab 1924 ist das alles weg; jetzt wird alles logisch konstruiert, und es gibt keine feststehende, fixe, unkonstruierte Unmittelbarkeit mehr. Dieser radikale Richtungswechsel war durch verschiedene Überlegungen motiviert, ${ }^{16}$ aber die wichtigste scheint mir Carnaps bewusste Distan-

\footnotetext{
${ }^{15}$ Auf diesen Einfluss wird in Carus (2007, S. 122-127, 148-150) näher eingegangen. Vaihinger war maßgeblich beeinflusst worden von der ersten Generation des Neukantianismus, vor allem von Helmholtz und (wie auch Nietzsche!) von F. A. Lange - was aber nicht heißt, dass er sich ganz unabhängig vom bekannteren amerikanischen Pragmatismus entwickelte; es scheint Berührungspunkte gegeben zu haben. Klaus Ceynowa (1993) zeigt, dass die proto-pragmatistischen Ideen des schottischen Philosophen und Psychologen Alexander Bain (ein Freund John Stuart Mills), die explizit von William James und Charles Peirce aufgegriffen wurden, auch über die Schriften des deutschen Neurophysiologen und Philosophen Adolf Horwicz auf Vaihinger einen von ihm selbst anerkannten bestimmenden Einfluss hatten.

${ }^{16}$ Hierzu Carus (2016), wo die oben zusammengefasste Rekonstruktion dieses Umbaus auch im Einzelnen belegt wird.
} 
zierung von der Ontologie zu sein, von der Forderung nach einem Endglied der sprachlichen Referenzkette. Mit anderen Worten: Für Carnap löste sich hier das Sprachliche von jeglicher Verantwortung gegenüber einer angeblichen „Realität“ in einer angeblich „wirklichen“ Welt. Carnap wandte die Husserlsche Einklammerungstechnik an, aber eben noch strenger als Husserl: Was eingeklammert wurde, war nicht nur die Außenwelt, auf die unsere Subjektivität transzendental hinzuweisen scheint, sondern auch die Subjektivität selbst. Nicht-eingeklammert blieb bei Carnap lediglich die Sprache selbst, eigentlich nur das Gerüst der Sprache, das reine Schema der logischen Strukturen, deren Sinn und Bedeutung wir eindeutig festlegen können, ohne uns von irrelevanten ererbten Assoziationen irreführen lassen zu müssen.

Was die drei Hauptpunkte miteinander verbindet, ist, dass sie zusammen einen weiteren Schritt in Richtung eines reineren Funktionalismus darstellen: Wenn der Unterschied zwischen Sprachbereichen aus ihren verschiedenen Funktionen entsteht, dann braucht man weder verschiedene Seinsarten noch Ontologie überhaupt. Damit kann auch der Gegenstandsbereich (also die Basis) ${ }^{17}$ einheitlich (,,monistisch“) sein, denn er hat nicht mehr den Anspruch, irgendeine letztendliche „,Realität" widerzuspiegeln oder zu repräsentieren. Und die Überwindung von Subjektivität heißt nicht, dass sie verneint oder trivialisiert wird, wie das damals im Behaviorismus und gegenwärtig bei Dennett und anderen manchmal getan wird, sondern bloß, dass sie völlig naturalistisch aufzufassen ist (also wieder: keine verschiedene Seinsart; dies ist genau der minimale „Subjektnaturalismus“ von Huw Price)..$^{18}$ Und ganz im Gegensatz zu Rickert oder Husserl, eher im Sinne von Helmholtz. Mit dieser zunehmenden Herauskristallisierung der funktionalistischen Gesamtansicht Carnaps verliert die Unterscheidung von Sein und Gelten (die vor 1924 durchaus eine Rolle gespielt haben mag) auch an Bedeutung.

\subsection{Aufbau (1928)}

Nun habe ich kurz - zu kurz - eine Entwicklung zwischen 1911 und 1924 skizziert, die mit Carnaps späterer Entwicklung nach 1928 sehr gut zusammenpasst: eine relativ geradlinige Entwicklung also zu einem globalen Funktionalismus hin, wie er zuerst im Toleranzprinzip von 1934 ganz explizit gemacht wird und erst im Spätwerk teilweise ausgeführt wurde. ${ }^{19}$

\footnotetext{
${ }^{17}$ Der „Bausteine“ (wie sie in „Vom Chaos zur Wirklichkeit“ heißen) oder der „Elementarerlebnisse" (im Aufbau).

${ }^{18}$ Siehe z. B. Price (2013), auch Carus (2018) und Price (2018).

${ }^{19}$ Diese relativ geradlinige Entwicklung habe ich ausführlicher in Carus (2007) dargestellt, obwohl die früheren Dokumente, die im gegenwärtigen Aufsatz herangezogen werden (vor allem die Freischar-Rede von 1911 und der offene Brief an Pastor LeSeur von 1917) dort noch nicht verwertet sind. Das im Buch aufgrund anderer Zeugnisse gezeichnete Bild wird durch diese allerdings weitgehend bestätigt.
} 
Vor diesem Hintergrund möchte ich nun das Wertekapitel (§ 152) im Aufbau noch einmal anschauen, um zu sehen, ob es diese geradlinige Entwicklung unterbricht, wie Mormann glaubt, oder einigermaßen in sie eingefügt werden kann.

Die Konstitution der Werte wird im § 152 nur sehr kursorisch skizziert. Obwohl Werte als „höhere geistige Gegenstände“ klassifiziert werden (die ansonsten wiederum auf die ,primären geistigen Gegenstände“ aufgebaut sind), nimmt die Konstitution der Werte eine ganz andere Form an; sie werden nicht aus anderen geistigen Gegenständen (auch nicht aus fremdpsychischen, psychischen oder physischen Gegenständen) konstituiert. Vielmehr greift ihre Konstitution auf eine viel niedrigere Stufe des Systems zurück, und zwar auf die Elementarerlebnisse selbst. Denn es heißt im $\S 152$, dass die Werte aus „Werterlebnissen“ konstituiert werden. Diese aber sind im Text des Buches vor § 152 kein einziges Mal erwähnt worden; man muss also annehmen, dass sie in den Elementarerlebnissen holistisch enthalten sind, wie die übrigen Erlebnisse (und Dinge usw.), die aus den Elementarerlebnissen konstituiert worden sind, und erst noch durch Quasi-Analyse konstruiert werden müssten.

Es heißt ja im Aufbau (§ 64): „Für die Basis wird kein Unterschied gemacht zwischen den Erlebnissen, die auf Grund späterer Konstitution als Wahrnehmung, Halluzination, Traum, usw. unterschieden werden" (S. 86). Es wird hier nicht ausdrücklich gesagt, aber ich sehe keinen Grund dagegen anzunehmen, dass auch kein Unterschied gemacht wird zwischen Erkenntnissen, Gefühlen, Neigungen und Abneigungen, usw. In „Vom Chaos zur Wirklichkeit“ wird von den „Bausteinen“ (den späteren „Elementarerlebnissen“ des Aufbau) gesagt: „Auch hier stehen wir noch vor der Lostrennung der Gefühlsbetonungen und Willensregungen" (ASP 081-05-01, S. 2) - was wohl auch im Aufbau hätte stehen können. Als „Werterlebnisse" könnte man also alle Aspekte der Elementarerlebnisse betrachten, die zum Treffen einer (praktischen) Entscheidung relevant sein könnten, aber nicht im Konstitutionssystem der Erkenntnis konstituiert sind (also nicht wahr oder falsch sein können).

Als Resultate der Quasianalyse sind Werte somit natürlich intersubjektiv (d. h. „objektiv“), wie auch alles andere, das innerhalb des Systems konstituiert wird. Es braucht also nicht unbedingt als Anklang an den südwestdeutschen Neukantianismus ausgelegt zu werden, dass Carnap von einer Objektivität der Werte spricht. Und ohne diese Einstufung als Symptom des Südwest-Neukantianismus heißt Objektivität von Werten vor allem noch keineswegs, dass Carnap seinen bisherigen Funktionalismus aufgegeben hätte. Man kann durchaus an die Objektivität der Werte in diesem Sinne glauben, ohne dass man sie als „kognitiv“ betrachtet, also ohne dass man sie der Wahrheit oder Falschheit für fähig hält. (Wie das z. B. bei Hare (1952) und beim späteren Carnap $(1963,2017)$ - unter vielen anderen - der Fall ist.)

Auch wenn Carnap bei seiner skizzierten Konstitution der Werte absichtlich auf Rickert anspielt, was ich nicht ausschließen möchte, würde ich eine solche Anspielung anders bewerten als Mormann, der aus der bloßen Tatsache der Anspielung einen eindeutigen Einfluss ableitet. Es ist klar, dass Carnap im Aufbau zeigen will, dass sein logisch-strukturelles Konstitutionssystem nicht nur viel Neues kann, sondern außerdem vielen potenziell noch wertvollen Aspekten der philosophischen 
Tradition gerecht wird. Zu diesen Aspekten rechnete Carnap auch manches, was er selbst zu dem Zeitpunkt nicht mehr besonders wertvoll fand, aber von manchem Leser vielleicht noch für wichtig gehalten werden mochte. Genau dies ist ja der Sinn der drei „Sprachen“ neben der symbolischen - dass nämlich der realistisch oder fiktionalistisch sympathisierende Leser möglichst nicht zu sehr am symbolischen Schriftbild Anstoß nimmt und empfänglich für die Gesamtperspektive bleibt, die das Buch eröffnen will.

Dass es Anspielungen auf Rickert gibt - wie auf Kant, Vaihinger, den Positivismus und die Phänomenologie - überrascht also nicht weiter. Carnap will eben zeigen, dass er mit seinem System alles kann, was die konnten (und noch viel mehr dazu). Im Falle von Rickert ist es aber schwer, der Versuchung zu widerstehen, über die Behauptung des Ebensogutseins hinaus eine (nur leicht verschleierte) Kritik zu vermuten - denn Rickert war, mehr als diese anderen Autoren, mit dem deutschen Historismus identifiziert, und das war ja genau die „Art der Geschichtsbetrachtung ... [die] noch bis heute starken Einfluß ausübt“" die unkritisch das Bestehende verherrlicht, wie Carnap in „Deutschlands Niederlage“ sagt. „Dass diese Auffassung nicht nur von Staatsmännern, sondern auch von einflussreichen Vertretern der Geisteswissenschaften verkündet worden ist und zum Teil noch heute verkündet wird, bedeutet eine besonders schwere Belastung unserer, der Geistigen, Schuldrechnung und unserer Verantwortung für die Zukunft" (S. 16 f.). Denn Carnap sieht diese Auffassung der Gelehrten als mitursächlich für den Krieg. „Die Geistesverfassung Europas, die den Weltkrieg unvermeidbar und dann seine Beendigung bisher unmöglich machte, hat ihren Hauptnährboden in Deutschland“ (S. 15). Wer sollte mit diesen harten Worten gemeint sein, wenn nicht die Vertreter des deutschen Historismus? - und Rickert befand sich ja unter den philosophischen Hauptvertretern dieser Schule! ${ }^{20}$

Der Nachkriegs-Carnap ist eben nicht mehr der begeisterte Rickert-Hörer von 1911; der Krieg hat ihn verändert. Er tendiert jetzt immer mehr zur politischen und geistigen Ernüchterung, zur „Neuen Sachlichkeit“, wie es vor allem Hans-Joachim Dahms (2004) überzeugend dargestellt hat. Man sollte diese Anspielungen auf Rickert also meiner Meinung nach so lesen, dass Carnap hier sagt: „Alles, was man an Rickert noch gut finden mag, kann ich in meinem System auch, und zwar ohne den ganzen reaktionären, kriegstreibenden deutschen Kram."

Carnaps Aneignung der neuen, ernüchterten Nachkriegskultur geschah nicht mit einem Ruck, sofort nach seinem Eintritt in die USPD oder nach Versailles, sondern ging graduell, etappenweise vor sich. Den Umbau des ganzen Aufbau-Systems im Jahre 1924 sollte man bestimmt mit diesem graduellen Prozess in Verbindung bringen. Leider wissen wir nicht aus früheren Notizen und Konzepten, wie Carnap vor 1925 die Werte konstituieren wollte. Es gibt aber einen Anhaltspunkt dafür, dass im früheren System diese Taktik des § 152, auf die Elementarerlebnisse zurückzugreifen, auch in anderen Fällen angewandt wurde. Und zwar gibt es in „Vom Chaos zur Wirklichkeit“ einen kurzen Abschnitt über die Konstitution der Psychologie, in dem

\footnotetext{
${ }^{20}$ Siehe z. B. Beiser (2011), Kap. 10.
} 
Carnap genau dasselbe tut. Das Zurückschrecken vor der Integration der Psychologie in die Naturwissenschaft, das sich hier ausdrückt, ist schon von Interesse. In Carnaps Argumenten dafür (nämlich dass bei dem gegenwärtigen Stand der psychologischen Wissenschaft eine solche Integration keineswegs gewährleistet ist) kann man, glaube ich, ein Echo auf Husserls „Philosophie als strenge Wissenschaft“ hören. Und die Trennung der psychologischen Konstitution von der Konstitution der verschiedenen „Wirklichkeiten“ deutet auch in die Richtung der „Doppelaspekt"-Auffassungen von Mach und Avenarius (Sommer 1985). So sieht man, dass der frühe Carnap trotz seines Russellschen Ausgangspunktes keineswegs so radikal von seiner Tradition (seinen Traditionen, sollte man besser sagen) loszulösen ist, wie das oft getan wird.

Was man bestimmt sagen kann, ist, dass der publizierte Aufbau aus verschiedenen Schichten besteht. In einer ersten und gröbsten Unterscheidung sollte man all das aussortieren, was aus der Zeit vor 1924 stammt. In diesen Topf würde ich werfen, was als ,überschlagbar“ bezeichnet ist, und dann noch einiges dazu, z. B. den Abschnitt $\S 152$. Carnap brauchte diese Teile, weil sie offene Themen des Buches behandelten; sie kennzeichneten den Anspruch des Buches in verschiedene Richtungen. Carnap wollte zeigen, dass Antworten auf bestimmte unumgehbare Fragen, die sein Ansatz aufwarf, zumindest denkbar seien, auch wenn sie nur sehr vorläufige Antworten waren, die er selbst nicht mehr so ernst nahm.

Bei der Psychologie hatte sich Carnap eine andere Strategie einfallen lassen als die radikale Zuflucht ganz hinunter zu den Elementarerlebnissen. Über Psychologie hatte er ja auch als Student Vorlesungen besucht und selbst an experimentellen Studien teilgenommen; er hatte damit den einschlägigen Erfahrungs- und Bildungshintergrund, um selbstständig zu denken. Bei den Werten fühlte er sich vielleicht nicht so sicher; dort hat er die alte Taktik (Zuflucht zu den Elementarerlebnissen) offenbar stehen lassen. Warum? Es scheint auf jeden Fall gegen das neue Prinzip der Einheit des Gegenstandsbereichs zu verstoßen, oder folgt ihm höchstens der Form nach, nicht ohne Schummelei. Aber: Wir wissen nicht, was in der Niederschrift von 1925 alles stand. Die Strategie des $§ 152$ ist vielleicht nur die Spitze eines Eisberges; nicht nur die Psychologie, auch andere Gebiete könnten wohl so konstituiert worden sein in der Zeit vor 1924, und hatten die Wende aus Zeitmangel überlebt. Die Alltagswelt der Dinge und Eigenschaften wurde in der ersten Fassung (1922) separat von der Welt der Physik konstituiert, nicht Physik aus Alltagswelt, wie im fertigen Aufbau (Carus 2016, S. 144). Wir können annehmen, dass die Fassung von 1925 sich zumindest in dieser Hinsicht dem publizierten Buch annäherte. Aber bei anderen Gebieten, z. B. der Psychologie, könnte ich mir vorstellen, dass erst Neurath sie beanstandete, als Carnap nach Wien gezogen war. Wir wissen eben noch zu wenig. 


\subsection{Archivalien}

Unveröffentlichte Manuskripte aus Carnaps Nachlass werden zitiert nach ihren Siglen in den Carnap Papers der Archives of Scientific Philosophy (abgekürzt ASP), wobei jeweils die erste Zahl vor dem Bindestrich die Kartonnummer, die zweite (zwischen den Bindestrichen) die Mappe innerhalb des Kartons, und die dritte die Seitenzahl innerhalb der Mappe bezeichnet.

\section{Literatur}

Awodey, S., und A. W. Carus. 2001. Carnap, completeness, and categoricity: The Gabelbarkeitssatz of 1928. Erkenntnis 54:145-572.

2007. Carnap's dream: Wittgenstein, Gödel, and Logical Syntax. Synthese 159:23-45.

2009. From Wittgenstein's prison to the boundless ocean: Carnap's dream of logical syn-

tax. In Carnap's Logical syntax of language, Hrsg. P. Wagner, 79-106. Basingstoke: Palgrave Macmillan.

Beiser, F. C. 2011. The German historicist tradition. Oxford: Oxford University Press.

Carnap, R. 1928. Der logische Aufbau der Welt. Berlin: Weltkreis.

1934. Theoretische Fragen und praktische Entscheidungen. Natur und Geist 2:257-260.

. 1963. Intellectual autobiography und Replies and systematic expositions. In Schilpp 1963, S. 1-84 und 859-1013.

2017. Value concepts. Synthese 194:185-194.

Carus, A. W. 2007. Carnap and twentieth-century thought: Explication as enlightenment. Cambridge: Cambridge University Press.

- 2016. Carnap and phenomenology: What happened in 1924? In Influences on the Aufbau, Hrsg. C. Damböck, 137-162. Wien: Springer.

- 2017. Carnapian rationality. Synthese 194:163-184.

2018. Going global: Carnap's voluntarism and Price's expressivism. Monist 101:441-467.

Ceynowa, K. 1993. Zwischen Pragmatismus und Fiktionalismus: Hans Vaihingers „Philosophie

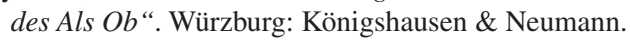

Creath, R. 1994. Functionalist theories of meaning and the defense of analyticity. In Logic, language, and the structure of scientific theories, Hrsg. W. Salmon und G. Wolters, 287-304. Pittsburgh: University of Pittsburgh Press.

Dahms, H.-J. 2004. Neue Sachlichkeit in the architecture and philosophy of the 1920s. In Carnap brought home: The view from Jena, Hrsg. S. Awodey und C. Klein, 353-372. LaSalle: Open Court.

Damböck, C., G. Sandner, und M. Werner, Hrsg. 2019. Logical empiricism, life reform, and the German youth movement/Logischer Empirismus, Lebensreform und die deutsche Jugendbewegung. Veröffentlichungen des Instituts Wiener Kreis. Dordrecht: Springer.

Hare, R. H. 1952. The Language of Morals (Oxford: Oxford Univerity Press).

Mormann, T. 2006. Werte bei Carnap. Z philos Forsch 60:169-189.

. 2010. Germany's defeat as a program: Carnap's philosophical and political beginnings. Unveröffentlicht, zugänglich über PhilPapers.

- 2016. Carnap's Aufbau in the Weimar context. In Influences on the Aufbau. Vienna Circle Institute Yearbook 18, Hrsg. C. Damböck, 115-136. Wien: Springer.

Price, H. 1988. Facts and the function of truth. Oxford: Blackwell.

. 1997. Naturalism and the fate of the M-worlds. Price 2011:132-147.
2011. Naturalism without mirrors. Oxford: Oxford University Press.

Price, H., et al. 2013. Expressivism, pragmatism, and representationalism. Cambridge: Cambridge University Press. 
Price, H. 2018. Carnapian voluntarism and global expressivism: Reply to Carus. Monist 101: 468-475.

Psillos, S. 2000. Rudolf Carnap's ,Theoretical concepts in science'. Studies in History and Philosophy of Science 31:151-172.

Richardson, A. 2007. Carnapian pragmatism. In The Cambridge companion to Carnap, Hrsg. M. Friedman und R. Creath, 295-315. Cambridge: Cambridge University Press.

Schilpp, P., Hrsg. 1963. The philosophy of Rudolf Carnap. LaSalle: Open Court.

Sommer, M. 1985. Husserl und der frühe Positivismus. Frankfurt a. M.: Klostermann.

Strawson, P. 1963. Carnap's views on constructed systems vs. natural languages in analytic philosophy. Schilpp 1963:503-518.

Werner, M. G. 2003. Moderne in der Provinz: Kulturelle Experimente im Fin de Siècle Jena. Göttingen: Wallstein.

- 2015. Freideutsche Jugend und Politik: Rudolf Carnaps Politische Rundbriefe 1918. In Geschichte intellektuell: Theoriegeschichtliche Perspektiven, Hrsg. F. W. Graf, E. Hanke und B. Picht, 465-486. Tübingen: Mohr Siebeck.

Williams, B. 1985. Ethics and the limits of philosophy. London: Fontana.

Open Access This chapter is licensed under the terms of the Creative Commons Attribution 4.0 International License (http://creativecommons.org/licenses/by/4.0/), which permits use, sharing, adaptation, distribution and reproduction in any medium or format, as long as you give appropriate credit to the original author(s) and the source, provide a link to the Creative Commons license and indicate if changes were made.

The images or other third party material in this chapter are included in the chapter's Creative Commons license, unless indicated otherwise in a credit line to the material. If material is not included in the chapter's Creative Commons license and your intended use is not permitted by statutory regulation or exceeds the permitted use, you will need to obtain permission directly from the copyright holder.

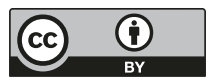

